\title{
On the Regularity Conditions for the Navier-Stokes and Related Equations
}

Dongho Chae

\begin{abstract}
We obtain a regularity conditions for solutions of the 3D NavierStokes equations with fractional powers of the Laplacian, which incorporates the vorticity direction and its magnitude simultaneously. We find that regularity assumption of direction field of the vorticity compensates with the integrability condition for the magnitude of vorticity. The regularity of direction field is most naturally measured in terms of the Triebel-Lizorkin type of norms. This unifies and extends previous results in this direction of studies, where the geometric structure of the vortex stretching term is used to obtain refined regularity conditions, initiated by Constantin and Fefferman.
\end{abstract}

\section{Introduction}

We are concerned with the following 'generalized' Navier-Stokes equations:

$$
\begin{aligned}
\frac{\partial v}{\partial t}+(v \cdot \nabla) v & =-\nabla p-\nu \Lambda^{\alpha} v \\
\operatorname{div} v & =0 \\
v(x, 0) & =v_{0}(x)
\end{aligned}
$$

where $v=\left(v^{1}, v^{2}, v^{3}\right), v^{j}=v^{j}(x, t), j=1,2,3$ is the velocity of the fluid flows, $p=p(x, t)$ is the scalar pressure, $v_{0}(x)$ is a given initial velocity field satisfying $\operatorname{div} v_{0}=0$, and $\nu>0$ is the viscosity constant. We are using the notation, $\Lambda^{\alpha}=(-\Delta)^{\frac{\alpha}{2}}$. We denote the system (1.1)-(1.3) by $(N S)_{\alpha}$. The case $\alpha=2$ corresponds to the usual Navier-Stokes equations, which we denote simply by $(N S)$. Heuristically, number $\alpha$ represents the 'strength of dissipation', and in this paper we are concerned with the case $0<\alpha \leq 2$.

2000 Mathematics Subject Classification: 35Q30, 76D03, 76D05.

Keywords: Navier-Stokes equations, regularity conditions, Triebel-Lizorkin type of spaces. 
We note that the system $(N S)_{\alpha}$ was first considered by J.L. Lions in [25], and the global regularity for $\alpha \geq \frac{5}{2}$ is shown there. We also remark that more recently there were studies on the small data global well-posedness on the same equation with $0 \leq \alpha \leq 2$ by the author of this paper, using the scale invariant Besov spaces ([14]) (see also [33, 7] for related studies). Taking curl of (1.1), we obtain the following vorticity equation.

$$
\frac{\partial \omega}{\partial t}+(v \cdot \nabla) \omega=(\omega \cdot \nabla) v-\nu \Lambda^{\alpha} \omega
$$

where the vorticity $\omega$ is defined by

$$
\omega=\operatorname{curl} v \text {. }
$$

The incompressibility condition (1.2) combined with (1.5) implies the BiotSavart's law,

$$
v(x, t)=-\frac{1}{4 \pi} \int_{\mathbb{R}^{3}} \frac{y \times \omega(x+y, t)}{|y|^{3}} d y
$$

for sufficiently rapidly decaying vorticity near infinity. After pioneering work by J. Leray ([24]) there are many comprehensive literatures on the existence theory of the weak solution of the $(N S)$ equations (say, $[23,31,26]$ ). The regularity of this weak solution is known as one of the most challenging problems in the mathematical fluid mechanics. In this note we are concerned with the regularity condition of the weak solutions of $(N S)_{\alpha}$. The first result for $(N S)$ in this direction is the one by Prodi ([27]), which states that if weak solution $v(x, t)$ satisfies

$$
v \in L^{r}\left(0, T ; L^{p}\left(\mathbb{R}^{3}\right)\right), \quad \frac{3}{p}+\frac{2}{r} \leq 1
$$

for $3<p \leq \infty$, then $v(x, t)$ is regular. After that there are further developments and refinements by Serrin ([29]), Fabes-Jones-Riviere ([20]), KozonoTaniuchi ([22]), and Escauriaza-Sverak-Seregin ([19]). In particular, Beirão da Veiga ([3]) obtained regularity condition in terms of $\nabla v$, which is equivalent to the one in terms of the vorticity due to the Calderon-Zygmund inequality. This states that if the vorticity, $\omega=\operatorname{curl} v$ of the weak solution $v$ satisfies

$$
\omega \in L^{r}\left(0, T ; L^{p}\left(\mathbb{R}^{3}\right)\right), \quad \frac{3}{p}+\frac{2}{r} \leq 2,
$$

for $\frac{3}{2}<p \leq \infty$, then $v$ becomes regular. This condition is later improved in [13], requiring the same regularity condition only for the two components of the vorticity. 
On the other hand, Constantin and Fefferman discovered remarkable geometric structures of the vortex stretching term, the first one of the right hand side of (1.4), which leads to the following statements [16] (see also [15]). Let $\xi(x, t)=\omega(x, t) /|\omega(x, t)|$ be the direction field of the vorticity, and let $\theta(x, y, t)$ be the angle between $\xi(x, t)$ and $\xi(x+y, t)$. If $\theta(x, y, t)$ satisfies

$$
|\sin \theta(x, y, t)| \leq C|y|
$$

in the region where the $|\omega(x, t)|,|\omega(x+y, t)|>K$ for some large constant $K$, then the solution becomes regular. After that this geometric structures have been used by many authors, say [17, 11, 10, 5, 2, 21]. For the convenience in the later discussion of our main result we describe here the results in [5] and [2]. In [5] it is proved that if

$$
|\sin \theta(x, y, t)| \leq g(x, t)|y|^{s}, \quad \text { for some function } \quad g \in L^{r}\left(0, T ; L^{p}\left(\mathbb{R}^{3}\right)\right)
$$

$$
\text { with } \frac{3}{p}+\frac{2}{r}=s-\frac{1}{2}
$$

for some $s \in[1 / 2,1], r \in\left[\frac{4}{2 s-1}, \infty\right]$ in the region where $|\omega(x, t)|,|\omega(x+y, t)|>K$ for some large constant $K$, then the solution becomes regular on $[0, T]$. We note here that for $p=r=\infty, s=1 / 2$ the condition (1.9) reduces to

$$
|\sin \theta(x, y, t)| \leq C|y|^{\frac{1}{2}}
$$

which shows genuine improvement of [5] from [16]. In order to handle the case $s \in(0,1 / 2]$ in $(1.9)$ the following is proved in [2]. If

$$
|\sin \theta(x, y, t)| \leq|y|^{s} \quad \text { and } \quad \omega \in L^{2}\left(0, T ; L^{p}\left(\mathbb{R}^{3}\right)\right), \quad \frac{3}{p}=s+1
$$

for some $s \in\left(0, \frac{1}{2}\right]$ in the region where the $|\omega(x, t)|,|\omega(x+y, t)|>K$ for some large constant $K$, then the solution becomes regular on $[0, T]$. We observe here that there is an extra condition of suitable integrability of the vorticity besides the regularity condition of the direction. Before stating our main theorems below we note that all of the above regularity conditions for weak solutions can be viewed as continuation principle for local in time strong solutions in $H^{s}\left(\mathbb{R}^{3}\right), s>5 / 2$. In the case considered in this paper, since the existence of weak solutions is not yet proved rigorously for $0<\alpha<2$, in particular (although I believe it could be done following the same lines of proof as the case of $\alpha=2$ ), the regularity conditions stated in Theorem 1.1 and Theorem 1.2 below should be understood as the continuation principle for local in time strong solution. We now state our main results in this paper. The main purpose of the following first result is to use it to prove Theorem 1.2 below, which we state as a theorem, since it is interesting in itself. 
Theorem 1.1 Let $\omega$ is the vorticity of a solution of $v$ for $(N S)_{\alpha}, 0<\alpha \leq 2$, satisfying

$$
\omega \in L^{r}\left(0, T ; L^{p}\left(\mathbb{R}^{3}\right)\right), \quad \text { with } \quad \frac{3}{p}+\frac{\alpha}{r} \leq \alpha,
$$

where $\frac{6}{\alpha}<p \leq \infty$. Then, there is no singularity up to $T$.

Remark 1.1: The above theorem says quantitatively that for the regularity of solutions of weaker dissipative term we need higher integrability of the vorticity. For $\alpha=2$ (1.11) reduces to (1.8). For $p=\infty, r=1$ (1.11) reduces to the Beale-Kato-Majda condition [1].

Remark 1.2: We observe that the system $(N S)_{\alpha}$ is invariant under scaling transform,

$$
v(x, t) \rightarrow v^{\lambda}(x, t)=\lambda^{\alpha-1} v\left(\lambda x, \lambda^{\alpha} t\right), \quad p(x, t) \rightarrow p^{\lambda}(x, t)=\lambda^{2 \alpha-2} p\left(\lambda x, \lambda^{\alpha} t\right),
$$

which induces the scaling for the vorticity, $\omega(x, t) \rightarrow \omega^{\lambda}(x, t)=\lambda^{\alpha} \omega\left(\lambda x, \lambda^{\alpha} t\right)$. For this scaling transform we have the norm invariance in the case of equality in (1.11) as follows.

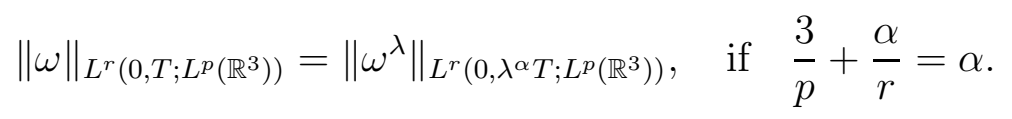

In this sense we can regard the condition (1.11) as optimal.

For the statement of our main result we introduce a function space. Given $0<s<1,1 \leq p \leq \infty, 1 \leq q \leq \infty$, the function space $\dot{\mathcal{F}}_{p, q}^{s}$ is defined by the seminorm,

$$
\|f\|_{\dot{\mathcal{F}}_{p, q}^{s}}=\left\{\begin{array}{c}
\left\|\left(\int_{\mathbb{R}^{n}} \frac{|f(x+y)-f(x)|^{q}}{|y|^{n+s q}} d y\right)^{\frac{1}{q}}\right\|_{L^{p}\left(\mathbb{R}^{n}, d x\right)} \text { if } 1 \leq p \leq \infty, 1 \leq q<\infty \\
\left\|{\operatorname{ess} \sup _{|y| \neq 0} \frac{|f(x+y)-f(x)|}{|y|^{s}}}_{\|}\right\|_{L^{p}\left(\mathbb{R}^{n}, d x\right)} \text { if } 1 \leq p \leq \infty, q=\infty
\end{array}\right.
$$

Observe that, in particular, $\dot{\mathcal{F}}_{\infty, \infty}^{s} \cong C^{s}$, the usual Hölder seminormed space. In order to compare this space with other more classical function spaces let us introduce the Banach space $\mathcal{F}_{p, q}^{s}$ by defining its norm,

$$
\|f\|_{\mathcal{F}_{p, q}^{s}}=\|f\|_{L^{p}}+\|f\|_{\dot{\mathcal{F}}_{p, q}^{s}} .
$$

We note that for $0<s<1,2 \leq p<\infty$ and $q=2, \mathcal{F}_{p, 2}^{s} \cong L_{s}^{p}\left(\mathbb{R}^{n}\right)=$ $(1-\Delta)^{-\frac{s}{2}} L^{p}\left(\mathbb{R}^{n}\right)$, the fractional order Sobolev space (or the Bessel potential space). (See [30, pp. 163]). 
If $\frac{n}{\min \{p, q\}}<s<1, n<p<\infty$ and $n<q \leq \infty$, then $\mathcal{F}_{p, q}^{s}$ coincides with the Triebel-Lizorkin space $F_{p, q}^{s}$ (See [32, pp. 101]). We note that there are previous papers (see $[6,8,9,11,12]$ ), where the the Triebel-Lizorkin space $F_{p, q}^{s}$ was used for the study of Cauchy problems in the fluid mechanics. The following is our main Theorem.

Theorem 1.2 Let $v(x, t)$ be a solution to $(N S)_{\alpha}$ and $\omega(x, t)=\operatorname{curl} v(x, t)$. Let $\xi(x, t)$ be its direction field, $\xi(x, t)=\omega(x, t) /|\omega(x, t)|$, defined for $\omega(x, t)$ $\neq 0$. Suppose there exists $s \in(0,1), q \in\left(\frac{3}{3-s}, \infty\right], p_{1} \in(1, \infty], p_{2} \in\left(1, \frac{3}{s}\right)$ satisfying $\frac{s}{3}<\frac{1}{p_{1}}+\frac{1}{p_{2}}<\frac{\alpha+s}{3}, \frac{1}{p_{2}}+\frac{1}{q}<1+\frac{s}{3}$ and $r_{1}, r_{2} \in[1, \infty]$ such that the followings hold.

$$
\begin{gathered}
\xi(x, t) \in L^{r_{1}}\left(0, T ; \dot{\mathcal{F}}_{p_{1}, q}^{s}\right) \quad \text { and } \omega(x, t) \in L^{r_{2}}\left(0, T ; L^{p_{2}}\left(\mathbb{R}^{3}\right)\right) \\
\text { with } \quad \frac{3}{p_{1}}+\frac{3}{p_{2}}+\frac{\alpha}{r_{1}}+\frac{\alpha}{r_{2}} \leq \alpha+s .
\end{gathered}
$$

Then, there is no singularity up to $T$.

Remark 1.3: Intuitively, the above theorem says that assumption of higher regularity of the direction vector field compensates with the assumption of the weaker integrability of the amplitude of vorticity field.

Remark 1.4: We consider the two special cases for $(N S)$ below $(\alpha=2$ case). First, let $p_{2}=r_{2}=2$. Then, we know that the Leray-Hopf weak solution $\omega$ satisfies $\int_{0}^{T}\|\omega(t)\|_{L^{2}}^{2} d t<\infty$, and the condition of the above theorem becomes

$$
\xi(x, t) \in L^{r_{1}}\left(0, T ; \dot{\mathcal{F}}_{p_{1}, q}^{s}\right), \quad \frac{3}{p_{1}}+\frac{2}{r_{1}} \leq s-\frac{1}{2} .
$$

Comparing this with (1.9), we find the natural identification of the function $g(x, t)$ as the direction field $\xi(x, t)$. Moreover, since we allow any finite number for $q$ in $\left(\frac{3}{3-s}, \infty\right]$, not necessarily infinity, the condition (1.13) is a generalization of (1.9).

Secondly, we observe that in the case $p_{1}=r_{1}=\infty$, and $s \in\left(0, \frac{1}{2}\right]$ the condition of the above theorem reduces to

$$
\xi \in \dot{\mathcal{F}}_{\infty, q}^{s}, \quad \omega \in L^{r_{2}}\left(0, T ; L^{p_{2}}\left(\mathbb{R}^{3}\right)\right) \quad \text { with } \quad \frac{3}{p_{2}}+\frac{2}{r_{2}} \leq s+2 .
$$

Since $\dot{\mathcal{F}}_{\infty, \infty}^{s} \cong C^{s}$, and $|\sin \theta(x, y, t)| \leq|\xi(x+y, t)-\xi(x, t)|$, as can be checked by elementary geometry, we have that

$$
|\sin \theta(x, y, t)| \leq C|y|^{s} \quad \text { if } \quad \xi \in L^{\infty}\left(0, T ; \dot{\mathcal{F}}_{\infty, \infty}^{s}\right) .
$$

Hence, we find that (1.10) is a special case for $q=\infty, r_{2}=2$ of (1.14). 


\section{Proof of the Main Theorems}

We first recall the following continuation principle (blow-up criterion) for the 3D Euler equations, due to Beale, Kato and Majda [1]:

Theorem 2.1 Suppose $v(x, t)$ is a local in time classical solution of the $3 D$ Euler equation, namely the system (1.1)-(1.3) with $\nu=0$, corresponding to initial data $v_{0} \in H^{s}\left(\mathbb{R}^{3}\right), s>\frac{5}{2}$. If we have a priori estimate for vorticity, $\int_{0}^{T}\|\omega(t)\|_{L^{\infty}} d t<\infty$, then we have $\lim \sup _{t \nearrow T}\|v(t)\|_{H^{s}}<\infty$, and in particular there is no singularity up to $T$.

In order to apply this theorem to our system $(N S)_{\alpha}$, we just observe that the exactly same continuation principle holds for our system for any $\alpha \in[0,2]$, which can be proved by obvious modifications of the proof in [1].

Prof of Theorem 1.1 Let $p_{1} \in[2, \infty)$ be of the form $p_{1}=2^{m}$, where $m$ is a positive integer. We take $D=\left(\partial_{x_{1}}, \partial_{x_{2}}, \partial_{x_{2}}\right)$ to (1.4), and then take inner product it with $D \omega|D \omega|^{p_{1}-2}$. After integration by part we have

$$
\begin{aligned}
& \frac{1}{p_{1}} \frac{d}{d t}\|D \omega\|_{L^{p_{1}}}^{p_{1}}+\nu \int_{\mathbb{R}^{3}}\left(\Lambda^{\alpha} D \omega\right) D \omega|D \omega|^{p_{1}-2} d x \\
& \quad=-\int_{\mathbb{R}^{3}} D[(v \cdot \nabla) \omega] \cdot D \omega|D \omega|^{p_{1}-2} d x+\int_{\mathbb{R}^{3}} D[(\omega \cdot \nabla) v] \cdot D \omega|D \omega|^{p_{1}-2} d x \\
& (2.1)=I+J .
\end{aligned}
$$

The viscosity term on the left hand side is estimated by

$$
\begin{gathered}
\nu \int_{\mathbb{R}^{3}}\left(\Lambda^{\alpha} D \omega\right) D \omega|D \omega|^{p_{1}-2} d x \geq \frac{\nu}{p_{1}} \int_{\mathbb{R}^{3}}\left|\Lambda^{\frac{\alpha}{2}}\left(|D \omega|^{\frac{p_{1}}{2}}\right)\right|^{2} d x \\
\geq \frac{\nu C_{\alpha}}{p_{1}}\left(\int_{\mathbb{R}^{3}}|D \omega|^{\frac{3 p_{1}}{3-\alpha}} d x\right)^{\frac{3-\alpha}{3}}=\frac{\nu C_{\alpha}}{p_{1}}\|D \omega\|_{L^{\frac{3 p_{1}}{3-\alpha}}}^{p_{1}}
\end{gathered}
$$

where we used Lemma 2.4 of [18] for the estimate of the fractional derivative in the first inequality (we note that the special form $p_{1}=2^{m}$ is used to apply this lemma), and used the Sobolev imbedding, $L_{\frac{\alpha}{2}}^{2}\left(\mathbb{R}^{3}\right) \hookrightarrow L^{\frac{6}{3-\alpha}}\left(\mathbb{R}^{3}\right)$ in the second inequality, where $L_{s}^{p}\left(\mathbb{R}^{n}\right)$ is the Bessel potential space introduced in the previous section before Theorem 1.2. Next, we estimate $I, J$ below.

$$
\begin{aligned}
I & =-\int_{\mathbb{R}^{3}} D v \cdot \nabla \omega \cdot D \omega|D \omega|^{p_{1}-2} d x-\int_{\mathbb{R}^{3}}(v \cdot \nabla) D \omega \cdot D \omega|D \omega|^{p_{1}-2} d x \\
& =I_{1}+I_{2} .
\end{aligned}
$$

Integrating by part, and using the fact, $\operatorname{div} v=0$, we obtain

$$
I_{2}=-\frac{1}{p_{1}} \int_{\mathbb{R}^{3}}(v \cdot \nabla)|D \omega|^{p_{1}} d x=\frac{1}{p_{1}} \int_{\mathbb{R}^{3}}(\operatorname{div} v)|D \omega|^{p_{1}} d x=0 .
$$


By Hölder's inequality and the standard $L^{p}$-interpolation inequality together with the Calderon-Zygmund inequality we estimate,

$$
\begin{aligned}
\left|I_{1}\right| & =\int_{\mathbb{R}^{3}}|D v\|D \omega\| D \omega|^{p_{1}-1} d x \leq\|D v\|_{L^{p}}\left\|_{L^{\frac{p p_{1}}{p-p_{1}}}}\right\|_{L^{p_{1}}}^{p_{1}-1} \\
& \leq C\|\omega\|_{L^{p}}\left\|_{L^{\frac{p_{1}}{\alpha p}}}^{1-\frac{3 p_{1}}{\alpha p_{1}}}\right\|_{L^{\frac{3 p_{1}}{3-\alpha}}}^{\frac{3 p^{2}}{\alpha^{p_{1}}}} \|_{L_{1}-1}^{p^{p_{1}}} \\
& =C\|\omega\|_{L^{p}}\left\|_{L^{p_{1}}}^{p_{1}-\frac{3 p_{1}}{\alpha p}}\right\|_{L^{\frac{3 p_{1}}{\alpha-\alpha}}}^{\frac{3 p_{1}}{3-\alpha}} .
\end{aligned}
$$

We note that the interpolation inequality used in here requires that $p_{1} \leq$ $\frac{p p_{1}}{p-p_{1}} \leq \frac{3 p_{1}}{3-\alpha}$, which is equivalent to $p_{1} \leq \frac{\alpha p}{3}$. Combined with $p_{1} \geq 2$, we have $p \geq 6 / \alpha$. In order to estimate $J$ we first decompose it into two terms as follows.

$$
\begin{aligned}
J & =\int_{\mathbb{R}^{3}} \cdot \nabla v \cdot\left\|^{p_{1}-2} d x+\int_{\mathbb{R}^{3}}(\omega \cdot \nabla) D v \cdot\right\|^{p_{1}-2} d x \\
& =J_{1}+J_{2} .
\end{aligned}
$$

Since

$$
J_{1} \leq \int_{\mathbb{R}^{3}}\|\nabla v\|^{p_{1}-1} d x
$$

the estimate of $J_{1}$ is the same as that of $I_{1}$. On the other hand, by the Hölder and the Calderon-Zygmund inequalities,

$$
J_{2} \leq\|\omega\|_{L^{p}}\left\|D^{2} v\right\|_{L^{\frac{p p_{1}}{p-p_{1}}}}\left\|_{L^{p_{1}}}^{p_{1}-1} \leq C\right\| \omega\left\|_{L^{p}}\right\|_{L^{\frac{p p_{1}}{p-p_{1}}}} \|_{L^{p_{1}}}^{p_{1}-1} .
$$

Hence, the estimate of $J_{2}$ is also the same as that of $I_{1}$. Combining the above estimates $I$, with (2.1)-(2.2), we have

$$
\begin{aligned}
\frac{d}{d t}\|\|_{L^{p_{1}}}^{p_{1}}+\nu C_{\alpha} \|_{L^{\frac{3 p_{1}}{3-\alpha}}}^{p_{1}} & \leq C\|\omega\|_{L^{p}}\left\|_{L^{p_{1}}}^{p_{1} \frac{3 p_{1}}{\alpha p}}\right\|_{L^{\frac{3 p_{1}}{3-\alpha}}}^{\frac{3 p_{1}}{\alpha p}} \\
& \leq C\|\omega\|_{L^{p}}^{\frac{\alpha p}{\alpha p^{p}-3}}\left\|_{L^{p_{1}}}^{p_{1}}+\frac{\nu C_{\alpha}}{2}\right\|_{L^{\frac{3 p_{1}}{3-\alpha}}}^{p_{1}}
\end{aligned}
$$

where we used Young's inequality, $a b \leq \varepsilon \frac{a^{u}}{u}+C_{\varepsilon} \frac{b^{u^{\prime}}}{u^{\prime}}, 1 / u+1 / u^{\prime}=1$. Absorbing the viscosity term to the left hand side, we find

$$
\frac{d}{d t}\left\|_{L^{p_{1}}}^{p_{1}}+\frac{\nu C_{\alpha}}{2}\right\|_{L^{\frac{3 p_{1}}{3-\alpha}}}^{p_{1}} \leq C\|\omega\|_{L^{p}}^{\frac{\alpha p}{\alpha p-3}} \|_{L^{p_{1}}}^{p_{1}} .
$$

Now, observe that the condition (1.11) is equivalent to

$$
r \geq \frac{\alpha p}{\alpha p-3}
$$


Hence, applying Gronwall's lemma and Hölder's inequality to (2.4), we obtain

$$
\begin{aligned}
\|(t)\|_{L^{p_{1}}} & \leq\left\|_{0}\right\|_{L^{p_{1}}} \exp \left[C \int_{0}^{T}\|\omega\|_{L^{p}}^{\frac{\alpha p}{\alpha p-3}} d t\right] \\
& \leq\left\|_{0}\right\|_{L^{p_{1}}} \exp \left[C\left(\int_{0}^{T}\|\omega\|_{L^{p}}^{r} d t\right)^{\frac{\alpha p}{r(\alpha p-3)}} T^{1-\frac{\alpha p}{r(\alpha p-3)}}\right] \\
& <\infty .
\end{aligned}
$$

for all $t \in[0, T]$, By the Gagliardo-Nirenberg inequality,

$$
\begin{aligned}
\|\nabla v(t)\|_{L^{\infty}} & \leq C\|v(t)\|_{L^{2}}^{\frac{2 p_{1}-6}{7 p_{1}-6}}\left\|D^{2} v(t)\right\|_{L^{p_{1}}}^{\frac{5 p_{1}}{7 p_{1}-6}} \leq C\left\|v_{0}\right\|_{L^{2}}^{\frac{2 p_{1}-6}{7 p_{1}-6}}\|D \omega(t)\|_{L^{p_{1}}}^{\frac{5 p_{1}}{7 p_{1}-6}} \\
& \leq C\left(\left\|v_{0}\right\|_{L^{2}},\left\|D \omega_{0}\right\|_{L^{p_{1}}}, T\right), \quad \forall t \in[0, T]
\end{aligned}
$$

and

$$
\int_{0}^{T}\|\omega(t)\|_{L^{\infty}} d t \leq \int_{0}^{T}\|\nabla v(t)\|_{L^{\infty}} d t \leq C T .
$$

We have shown that $\omega$ satisfies the Beale-Kato-Majda criterion, and hence we have regularity up to $T$.

Note after the proof. After finishing the paper the author was informed that the estimate (2.2) with $\alpha=2$ is obtained in [4]. The case $0<\alpha<2$, however, is much more delicate, and could be successfully handled thanks to Lemma 2.4 of [18] as described above by assuming integer power of $p_{1}$.

Proof of Theorem 1.2 Let $p$ be of the form $p=2^{m}, m \in \mathbb{N}$, and satisfy

$$
\frac{3}{\alpha} \leq p<\infty
$$

Taking $L^{2}\left(\mathbb{R}^{3}\right)$ inner product of $(1.4)$ by $\omega(x, t)|\omega(x, t)|^{p-2}$ and substituting $v$ from (1.6) into it, we have after integration by parts

$$
\begin{aligned}
& \frac{1}{p} \frac{d}{d t}\|\omega(t)\|_{L^{p}}^{p}+\nu \int_{\mathbb{R}^{3}}\left(\Lambda^{\alpha} \omega\right) \cdot \omega|\omega|^{p-2} d x \\
& \quad=\frac{3}{4 \pi} \int_{\mathbb{R}^{3}} \int_{\mathbb{R}^{3}}(\omega(x, t) \cdot \hat{y})(\omega(x+y, t) \times \omega(x, t) \cdot \hat{y}) \frac{d y}{|y|^{3}}|\omega(x, t)|^{p-2} d x \\
& (2.6) \quad=I,
\end{aligned}
$$

where the integral with respect to $y$ in the right hand side is in the sense of principal value. Here we used the notation, $\hat{y}=y /|y|$ for $y \in \mathbb{R}^{3}, y \neq 0$. 
We first estimate the viscosity term in the left hand side as follows.

$$
\begin{aligned}
\nu \int_{\mathbb{R}^{3}}\left(\Lambda^{\alpha} \omega\right) \cdot \omega|\omega|^{p-2} d x \geq \frac{\nu}{p} \int_{\mathbb{R}^{3}}\left|\Lambda^{\frac{\alpha}{2}}\left(|\omega|^{\frac{p}{2}}\right)\right|^{2} d x \\
\geq \frac{\nu C_{\alpha}}{p}\left(\int_{\mathbb{R}^{3}}|\omega|^{\frac{3 p}{3-\alpha}} d x\right)^{\frac{3-\alpha}{3}}=\frac{\nu C_{\alpha}}{p}\|\omega\|_{L^{\frac{3 p}{3-\alpha}}}^{p}
\end{aligned}
$$

which is similar to the estimates in (2.2) of the previous proof. Note that the assumption $p=2^{m}$ is used here as previously (see lines after (2.2)). Next, using the fact, $\xi(x, t) \times \xi(x, t)=0$, we estimate the vortex stretching term as follows.

$$
\begin{aligned}
& I=\frac{3}{4 \pi} \int_{\mathbb{R}^{3}} \int_{\mathbb{R}^{3}}(\xi(x, t) \cdot \hat{y})(\xi(x+y, t) \times \xi(x, t) \cdot \hat{y})|\omega(x+y, t)| \frac{d y}{|y|^{3}}|\omega(x, t)|^{p} d x \\
&=\frac{3}{4 \pi} \int_{\mathbb{R}^{3}} \int_{\mathbb{R}^{3}}(\xi(x, t) \cdot \hat{y})[(\xi(x+y, t)-\xi(x, t)) \times \xi(x, t) \cdot \hat{y}] \\
& \times|\omega(x+y, t)| \frac{d y}{|y|^{3}}|\omega(x, t)|^{p} d x \\
& \leq \frac{3}{4 \pi} \int_{\mathbb{R}^{3}} \int_{\mathbb{R}^{3}}|\xi(x+y, t)-\xi(x, t)||\omega(x+y, t)| \frac{d y}{|y|^{3}}|\omega(x, t)|^{p} d x \\
& \leq \frac{3}{4 \pi} \int_{\mathbb{R}^{3}}\left(\int_{\mathbb{R}^{3}} \frac{|\xi(x+y, t)-\xi(x, t)|^{q}}{|y|^{3+s q}} d y\right)^{\frac{1}{q}} \times\left(\int_{\mathbb{R}^{3}} \frac{|\omega(x+y, t)|^{q^{\prime}}}{|y|^{3-s q^{\prime}}} d y\right)^{\frac{1}{q^{\prime}}}|\omega(x, t)|^{p} d x \\
& \text { (2.8) } \quad \leq \frac{3}{4 \pi}\|\xi\|_{\dot{\mathcal{F}}_{p_{1}, q}^{s}}\left\|\left\{I_{s q^{\prime}}\left(|\omega|^{q^{\prime}}\right)\right\}^{\frac{1}{q^{\prime}}}\right\|\left\|_{L^{\tilde{p}_{2}}}\right\| \omega \|_{L^{p_{3}}}^{p},
\end{aligned}
$$

where

$$
\frac{1}{p_{1}}+\frac{1}{\tilde{p}_{2}}+\frac{p}{p_{3}}=1, \quad \frac{1}{q}+\frac{1}{q^{\prime}}=1,
$$

and $I_{\sigma}(\cdot), 0<\sigma<3$, is the operator defined by the Riesz potential as follows.

$$
I_{\sigma}(f)(x)=\gamma(\sigma) \int_{\mathbb{R}^{3}} \frac{f(x+y)}{|y|^{3-\sigma}} d y, \quad \gamma(\sigma)=2^{\sigma} \pi^{\frac{3}{2}} \frac{\Gamma\left(\frac{\sigma}{2}\right)}{\Gamma\left(\frac{3-\sigma}{2}\right)} .
$$

From the well-defined property of the Riesz operator we have the restriction $0<s q^{\prime}<3$, which gives us $q \in\left(\frac{3}{3-s}, \infty\right]$ due the second equation of $(2.9)$. 
Using the Hardy-Littlewood-Sobolev inequality ([30]), we estimate

$$
\begin{aligned}
\left\|\left\{I_{s q^{\prime}}\left(|\omega| q^{q^{\prime}}\right)\right\}^{\frac{1}{q^{\prime}}}\right\|_{L^{\tilde{p}_{2}}} & =\left\|I_{s q^{\prime}}\left(|\omega|^{q^{\prime}}\right)\right\|_{L^{\frac{\tilde{p}_{2}}{q^{\prime}}}}^{\frac{1}{q^{\prime}}} \\
& \leq C\left\||\omega|^{q^{\prime}}\right\|_{L^{r}}^{\frac{1}{q^{\prime}}}=C\|\omega\|_{L^{r q^{\prime}}}=C\|\omega\|_{L^{\frac{3 \tilde{p}_{2}}{3+s p_{2}}}},
\end{aligned}
$$

where we used the relation, $\frac{1}{r}=\frac{q^{\prime}}{\tilde{p}_{2}}+\frac{s q^{\prime}}{3}$, and hence $r q^{\prime}=\frac{3 \tilde{p}_{2}}{3+s \tilde{p}_{2}}$. Observe that we require here that $\tilde{p}_{2}>q^{\prime}$. On the other hand, using the standard $L^{p}$-interpolation inequality, we estimate

$$
\begin{aligned}
\|\omega\|_{L^{p_{3}}}^{p} & \leq C\|\omega\|_{L^{p}}^{\left.\frac{3 p}{p_{3}}-\frac{3}{\alpha}+1\right) p}\|\omega\|_{L^{\frac{3 p}{3-\alpha}}}^{\frac{3}{\alpha}-\frac{3 p}{\alpha p_{3}}} \\
& =C\|\omega\|_{L^{p}}^{p-\frac{3 p}{\alpha p_{1}}-\frac{3 p}{\alpha \tilde{p}_{2}}}\|\omega\|_{L^{\frac{3 p}{3-\alpha}}}^{\frac{3 p}{\alpha p_{1}}+\frac{3 p}{\alpha \tilde{p}_{2}}}
\end{aligned}
$$

where we used the relation (2.9). Note that use of the interpolation inequality in (2.11) requires that $p<p_{3}<\frac{3 p}{3-\alpha}$, which, in turn, gives us the condition

$$
0<\frac{1}{p_{1}}+\frac{1}{\tilde{p}_{2}}<\frac{\alpha}{3}
$$

due the first equation of (2.9). Combining (2.8) with (2.10) and (2.11), we derive

$$
\begin{aligned}
I & \leq C\|\xi\|_{\dot{\mathcal{F}}_{p_{1}, q}^{s}}\|\omega\|_{L^{\frac{3 \tilde{p}_{2}}{3+s p_{2}}}}\|\omega\|_{L^{p}}^{p-\frac{3 p}{\alpha p_{1}}-\frac{3 p}{\alpha \tilde{p}_{2}}}\|\omega\|_{L^{\frac{3 p}{3-\alpha}}}^{\frac{3 p}{\alpha p_{1}}+\frac{3 p}{\alpha \tilde{p}_{2}}} \\
& \leq C\left(\|\xi\|_{\dot{\mathcal{F}}_{p_{1}, q}^{s}}\|\omega\|_{L^{\frac{3 \tilde{p}_{2}}{3+s \tilde{p}_{2}}}}\right)^{\frac{\alpha p_{1} \tilde{p}_{2}}{\alpha p_{1} \tilde{p}_{2}-3 p_{1}-3 \tilde{p}_{2}}}\|\omega\|_{L^{p}}^{p}+\frac{\nu C_{\alpha}}{2 p}\|\omega\|_{L^{3-\alpha}}^{p}
\end{aligned}
$$

where we used Young's inequality, $a b \leq C_{\varepsilon} \frac{a^{u}}{u}+\varepsilon \frac{b^{u^{\prime}}}{u^{\prime}}$ with

$$
a=\|\xi\|_{\dot{\mathcal{F}}_{p_{1}, q}^{s}}\|\omega\|_{L^{\frac{3 \tilde{p}_{2}}{3+s \bar{p}_{2}}}}\|\omega\|_{L^{p}}^{p-\frac{3 p}{\alpha p_{1}}-\frac{3 p}{\alpha \tilde{p}_{2}}}, \quad b=\|\omega\|_{L^{\frac{3 p}{3-\alpha}}}^{\frac{3 p}{\alpha p_{1}}+\frac{3 p}{\alpha \tilde{p}_{2}}},
$$

and

$$
u=\frac{\alpha p_{1} \tilde{p}_{2}}{\alpha p_{1} \tilde{p}_{2}-3\left(p_{1}+\tilde{p}_{2}\right)}, \quad u^{\prime}=\frac{\alpha p_{1} \tilde{p}_{2}}{3\left(p_{1}+\tilde{p}_{2}\right)} .
$$

Setting $\frac{3 \tilde{p}_{2}}{3+s \tilde{p}_{2}}=p_{2}$, we have $\tilde{p}_{2}=\frac{3 p_{2}}{3-s p_{2}}$. We observe here that there is a restriction of $p_{2}<\frac{3}{s}$ due to positiveness of $\tilde{p}_{2}$. Combining this equality with the previous condition, $\tilde{p}_{2}>q^{\prime}$ (see lines after $(2.10)$ ), we also have $3 p_{2}>\left(3-s p_{2}\right) q^{\prime}$, which implies that

$$
\frac{1}{p_{2}}+\frac{1}{q}<1+\frac{s}{3}
$$


Substituting the value of $\tilde{p}_{2}$ into (2.13), we obtain

$$
I \leq C\|\xi\|_{\dot{\mathcal{F}}_{p_{1}, q}^{s}}^{Q}\|\omega\|_{L^{p_{2}}}^{Q}\|\omega\|_{L^{p}}^{p}+\frac{\nu C_{\alpha}}{2 p}\|\omega\|_{L^{3-\alpha}}^{p}
$$

where we set

$$
Q=\frac{\alpha p_{1} p_{2}}{(\alpha+s) p_{1} p_{2}-3 p_{1}-3 p_{2}} .
$$

We note that the restriction (2.12) becomes

$$
\frac{s}{3}<\frac{1}{p_{1}}+\frac{1}{p_{2}}<\frac{\alpha}{3}+\frac{s}{3}
$$

in terms of $p_{1}, p_{2}$. Substituting the estimates (2.15) and (2.7) into (2.6), and absorbing the viscosity term, $\frac{\nu C_{\alpha}}{2 p}\|\omega\|_{L^{\frac{3 p}{3-\alpha}}}^{p}$ to the left hand side, we have

$$
\frac{d}{d t}\|\omega(t)\|_{L^{p}}^{p}+\frac{\nu C_{\alpha}}{2}\|\omega(t)\|_{L^{3-\alpha}}^{p} \leq C\|\xi(t)\|_{\dot{\mathcal{F}}_{p_{1}, q}^{s}}^{Q}\|\omega(t)\|_{L^{p_{2}}}^{Q}\|\omega\|_{L^{p}}^{p}
$$

Now the condition (1.12) becomes

$$
\frac{1}{r_{1}}+\frac{1}{r_{2}} \leq \frac{1}{Q}
$$

in terms of $Q$. The Gronwall lemma applied to (2.17) combined with Hölder's inequality provides us with

$$
\begin{aligned}
& \|\omega(t)\|_{L^{p}} \leq\left\|\omega_{0}\right\|_{L^{p}} \exp \left[C \int_{0}^{T}\|\xi(t)\|_{\mathcal{F}_{p_{1}, q}^{s}}^{Q}\|\omega(t)\|_{L^{p_{2}}}^{Q} d t\right] \\
& \leq\left\|\omega_{0}\right\|_{L^{p}} \exp \left[\left(C \int_{0}^{T}\|\xi(t)\|_{\dot{\mathcal{F}}_{p_{1}, q}^{s}}^{r_{1}} d t\right)^{\frac{Q}{r_{1}}}\left(\int_{0}^{T}\|\omega(t)\|_{L^{p_{2}}}^{r_{2}} d t\right)^{\frac{Q}{r_{2}}} T^{\left(1-\frac{Q}{r_{1}}-\frac{Q}{r_{2}}\right)}\right]
\end{aligned}
$$

for all $t \in[0, T]$. Hence, $\omega \in L^{\infty}\left(0, T ; L^{p}\left(\mathbb{R}^{2}\right)\right)$. Integrating (2.17) over $[0, T]$, we have

$$
\begin{aligned}
& \|\omega(t)\|_{L^{p}}^{p}+\frac{\nu C_{\alpha}}{2} \int_{0}^{T}\|\omega(t)\|_{L^{\frac{3 p}{3-\alpha}}}^{p} d t \\
& \quad \leq C \sup _{0 \leq t \leq T}\|\omega(t)\|_{L^{p}}^{p} \int_{0}^{T}\|\xi(t)\|_{\dot{\mathcal{F}}_{p_{1}, q}^{s}}^{Q}\|\omega(t)\|_{L^{p_{2}}}^{Q} d t+\left\|\omega_{0}\right\|_{L^{p}}^{p}<\infty
\end{aligned}
$$

for all $t \in[0, T]$, and hence

$$
\int_{0}^{T}\|\omega(t)\|_{L^{3-\alpha}}^{p} d t<\infty .
$$


Since $\omega \in L^{p}\left(0, T ; L^{\frac{3 p}{3-\alpha}}\left(\mathbb{R}^{3}\right)\right)$, and our choice of $p$ in $(2.5)$ implies

$$
\frac{3}{r}+\frac{\alpha}{p} \leq \alpha \quad \text { with } \quad r=\frac{3 p}{3-\alpha}
$$

which is a special case of the condition in (1.11). Hence, applying Theorem 1.1, we find that the solution $v(x, t)$ is regular up to $T$.

\section{References}

[1] Beale, J. T., Kato, T. and Majda, A.: Remarks on the breakdown of smooth solutions for the 3-D Euler equations. Comm. Math. Phys. 94 (1984), 61-66.

[2] BeIRÃo DA VeIgA, H.: Vorticity and smoothness in incompressible viscous flows. In Wave Phenomena and Asymptotic Analysis, 37-42. RIMS Kōkyūroku 1315. Research Institute of Mathematical Sciences, Kyoto University, 2003.

[3] Beirão da Veiga, H.: Concerning the regularity problem for the solutions of the Navier-Stokes equations. C. R. Acad. Sci. Paris Sér. I Math. 321 (1995), no. 4, 405-408.

[4] Beirão Da Veiga, H.: Existence and asymptotic behavior for strong solutions of the Navier-Stokes equations in the whole space. Indiana Univ. Math. J. 36 (1987), no. 1, 149-166.

[5] Beirão da Veiga, H. And Berselli, L. C.: On the regularizing effect of the vorticity direction in incompressible viscous flows. Differential Integral Equations 15 (2002), no. 3, 345-356.

[6] Cannone, M.: Ondelettes, paraproduits et Navier-Stokes. Diderot Editeur, Paris, 1995.

[7] Cannone, M. and Karch, G.: Incompressible Navier-Stokes equations in abstract Banach spaces. In Tosio Kato's method and principle for evolution equations in mathematical physics (Sapporo, 2001), 27-41. Sūrikaisekikenkyūsho Kōkyūroku 1234, 2001.

[8] Cannone, M. and Planchon, F.: On the nonstationary Navier-Stokes equations with an external force. Adv. Differential Equations 4 (1999), 697-730.

[9] Cannone, M. and Planchon, F.: On the regularity of the bilinear term for solutions to the incompressible Navier-Stokes equations. Rev. Mat. Iberoamericana 16 (2000), 1-16.

[10] Chat, D.: Remarks on the blow-up criterion of the three-dimensional Euler equations. Nonlinearity 18 (2005), 1021-1029.

[11] Chat, D.: On the Euler equations in the critical Triebel-Lizorkin spaces. Arch. Ration. Mech. Anal. 170 (2003), no. 3, 185-210 
[12] Chat, D.: On the well-posedness of the Euler equations in the TriebelLizorkin spaces. Comm. Pure Appl. Math. 55 (2002), 654-678.

[13] Chae, D. and Choe, H.-J.: Regularity of solutions to the Navier-Stokes equations. Electron. J. Differential Equations 1999, no. 5, 7 pp. (electronic).

[14] Chae, D. And Lee, J.: On the global well-posedness and stability of the Navier-Stokes and the related equations. In Contributions to current challenges in mathematical fluid mechanics, 31-51. Adv. Math. Fluid Mech. Birkhäuser, Basel, 2004.

[15] Constantin, P.: Geometric statistics in turbulence. SIAM Rev. 36 (1994), $73-98$.

[16] Constantin, P. and Fefferman, C.: Direction of vorticity and the problem of global regularity for the Navier-Stokes equations. Indiana Univ. Math. J. 42 (1993), 775-789.

[17] Constantin, P., Fefferman, C. and Majda, A.: Geometric constraints on potentially singular solutions for the 3-D Euler equations. Comm. Partial Differential Equations 21 (1996), 559-571.

[18] Córdoba, A. And Córdoba, D.: A maximum principle applied to quasigeostrophic equations. Comm. Math. Phys. 249 (2004), no. 3, 511-528.

[19] Escauriaza, L., Seregin, G. and Shverak, V.: $L_{3, \infty}$-solutions of Navier-Stokes equations and backward uniqueness. Russian Math. Surveys 58 (2003), no. 2, 211-250.

[20] Fabes, E., Jones, B. And Riviere, N.: The initial value problem for the Navier-Stokes equations with data in $L^{p}$. Arch. Rational. Mech. Anal. 45 (1972), 222-240.

[21] Grujic, Z. and Ruzmaikina, A.: Interpolation between algebraic and geometric conditions for smoothness of the vorticity in the 3D NSE. Indiana Univ. Math. J. 53 (2004), no. 4, 1073-1080.

[22] Kozono, H. And Taniuchi, Y.: Bilinear estimates in BMO and the Navier-Stokes equations. Math. Z. 235 (2000), no. 1, 173-194.

[23] LadyžHenskaya, O. A.: The mathematical theory of viscous incompressible flow. Gordon and Breach, New York, 1969.

[24] Leray, J.: Essai sur le mouvement d'un fluide visqueux emplissant l'espace. Acta Math. 63 (1934), 193-248.

[25] Lions, J. L.: Quelques méthods de résolution des problémes aux limites nonlinéaires. Dunod, Paris, 1969.

[26] Majda, A. And Bertozzi, A.: Vorticity and incompressible flow. Cambridge Texts in Applied Mathematics 27. Cambridge Universitiy Press, Cambridge, 2002.

[27] Prodi, G.: Un Teorema di unicità per le equazioni di Navier-Stokes. Ann. Mat. Pura Appl. (4) 48 (1959), 173-182. 
[28] Ruzmaikina, A. And Grujić, Z.: On depletion of the vortex-stretching term in the 3D Navier-Stokes equations. Comm. Math. Phys. 247 (2004), 601-611.

[29] Serrin, J.: On the interior regularity of weak solutions of the NavierStokes equations. Arch. Rational Mech. Anal. 9 (1962), 187-195.

[30] Stein, E. M.: Singular integrals and differentiability properties of functions. Princeton Mathematical Series 30. Princeton University Press, Princeton, N. J., 1970.

[31] Temam, R.: Navier-Stokes equations. Theory and numerical analysis. Third edition. Studies in Mathematics and its Applications 2. NorthHolland Publishing Co., Amsterdam, 1984.

[32] Triebel, H.: Theory of function spaces. Monographs in Mathematics 78. Birkhäuser Verlag, Basel, 1983.

[33] Wu, J.: The generalized incompressible Navier-Stokes equations in Besov spaces. Dyn. Partial Differ. Equ. 1 (2004), 381-400.

Recibido: 7 de septiembre de 2005

Revisado: 7 de noviembre de 2005

Dongho Chae

Department of Mathematics

Sungkyunkwan University

Suwon 440-746, Korea

chae@skku.edu

This work was supported by Korea Research Foundation Grant (MOEHRD, Basic Research Promotion Fund). 